\title{
Comparison of the Imaging Manifestations and Diagnostic Values of Multi-slice Spiral CT and Enhanced MRI Scans of Primary Liver Cancer (PLC) Intrahepatic Lesions
}

\author{
Heng Tang ${ }^{*}$, Xiang Gao \\ Hubei No.3 People's Hospital of Jianghan University, Wuhan 430033, Hubei Province, China
}

\begin{abstract}
Objective: To compare and analyze the clinical diagnostic value of multi-slice spiral CT and enhanced MRI for primary liver cancer (PLC), and to summarize the imaging findings. Methods: The research subjects in this article were 50 patients with PLC who were admitted to our hospital from 2017 April to 2018 September. After the patients were admitted, they were examined by multi-slice spiral CT and enhanced MRI scanning, and the pathological diagnosis results were followed up. Relevant information was reviewed and analyzed. Results: The detection rate of multi-slice spiral CT was higher than that of MRI in the portal venous phase lesion detection. The difference was statistically significant $(P<0.05)$. The detection rate of lesions in the arterial phase and delayed phase was not statistically significant $(P>0.05)$; the diagnostic accuracy of multi-slice spiral CT was $85.96 \%$, and the diagnostic accuracy of enhanced MRI scanning was $91.11 \%$, which was not statistically significant $(P>0.05)$. MRI scans showed the capsules of primary liver cancer better than CT, but the difference was not statistically significant $(P>0.05)$. Conclusion: In conclusion, both multi-slice spiral CT and enhanced MRI can be used for the effective diagnosis of primary liver cancer intrahepatic lesions. The diagnostic value of the two is equivalent, but enhanced MRI has a slightly higher diagnostic accuracy and can be used as the preferred method.
\end{abstract}

Keywords: Multi-slice spiral CT; Enhanced MRI; Primary liver cancer; Imaging manifestations of the lesion; Diagnostic value
Publication date: November, 2020

Publication online: 30 November, 2020

"Corresponding author: Heng Tang, 358282436@ qq.com

As a common malignant tumor, primary liver cancer specifically refers to malignant tumors that occur in stem cells or intrahepatic bile duct epithelial cells. As the disease progresses, it can cause greater harm to the human body. Early diagnosis, early detection, and early treatment can improve the therapeutic effects of primary liver cancer and are of great significance to prolonging the survival time of patients ${ }^{[1]}$. Previous studies have confirmed that there is a close relationship between the occurrence of liver cancer and liver cirrhosis. About $5 \%$ of patients with liver cirrhosis will develop secondary liver cancer. Among patients with primary liver cancer, as high as about $70 \%$ of patients were found with liver cirrhosis. Through imaging examinations, liver spaceoccupying lesions in patients with cirrhosis can be found early and accurately, so as to provide reference and guidance for clinical diagnosis and treatment. Currently, in clinical practice, multi-slice spiral CT enhanced scanning and enhanced magnetic resonance imaging (MRI) scanning are two commonly used methods, and each has its diagnostic advantages and disadvantages. In order to clarify the application value of multi-slice spiral $\mathrm{CT}$ and enhanced MRI scanning in the diagnosis of PLC liver lesions to provide intervention for the early treatment of PLC patients, this paper combines the 50 patients with PLC admitted to our hospital during 2017 April 2018 September to discuss the diagnostic value of 
multi-splice spiral CT and enhanced MRI scan, the detailed analysis is as follows:

\section{Information and Methods}

\subsection{General Information}

The research subjects in this paper were 50 patients with PLC admitted to our hospital from 2017 April to 2018 September. All patients were finally diagnosed as hepatocellular carcinoma through histopathology. Among them, 29 were male patients and 21 were female patients, ranging in age from 33 to 79 years old, the average age was $(55.91 \pm 4.74)$ years, ChildPugh classification: 23 cases of grade A, 17 cases of grade B, 10 cases of grade $\mathrm{C}$. All patients underwent multi-slice spiral CT and enhanced MRI scans. Meanwhile, there was no history of anti-tumor treatment. The examination data were preserved. The research was approved by the ethics committee of our hospital. Exclusion criteria: (1) Patients with circulatory system diseases, or history of blood transfusion in the past 30 days; (2) Abdominal trauma history, previous history of abdominal surgery; (3) Pregnant and lactating women; (4) Mental disorders; (5) Patients with other malignant tumors, such as germline embryonic tumors or metastatic liver cancer.

\subsection{Methods}

Equipment: Philips Brilliance 16-slice spiral CT for CT scanning; Discovery MR750w 3.0T nuclear magnetic resonance instrument produced by GE in the United States for MRI.

Multi-slice spiral CT examination: Before the examination, inform the patient of the main points of cooperation. Complete the examination in the supine position. The examination area is from the top of the diaphragm to the lower edge of the liver. The conventional $\mathrm{CT}$ examination was completed according to the conventional method, and then the dynamic enhanced scan was performed. Ion-contrast agent iohexol, the arterial phase examination was performed 25-30s after injection, the portal venous phase examination was performed during 50-60s, and the delayed phase scanning was performed during 120-180s. The acquired image data was sent to the corresponding workstation to complete the examination.

MRI enhanced scanning examination: the same health education before the examination, inform the patients of the precautions. The 8-channel TORSPA phased array was selected for the surface coil, the examination was completed in the supine position, and the artery scan was performed after the conventional plain scan. The dynamic scan uses LAVA technology, where the layer thickness = $4.4 \mathrm{~mm}$, matrix $=272 \times 160, \mathrm{TE}=1.2 \mathrm{~ms}, \mathrm{TR}=2.6$ $\mathrm{ms}$, reversal angle $=15^{\circ}$, time $=5 \mathrm{~ms}$, number of excitations $=0.73$, field of view $=36 \times 40 \mathrm{~cm}$, breath holding time $21 \mathrm{~s}$. After setting the parameters, a whole liver volume scan was performed, contrast medium was injected, and arterial phase examination was performed at 17-20s after injection, portal venous phase examination at $60-63 \mathrm{~s}$, and the delayed phase scan was done after $180 \mathrm{~s}$.

All CT and MRI examination data were randomly divided into two types by 4 clinicians, and they made diagnoses without knowing each other, and reached a consensus through exchange of opinions to make a disease diagnosis.

\subsection{Observation Indicators}

Observation indicators: (1) pathological diagnosis results; (2) lesion detection rate; (3) diagnosis accuracy; (4) imaging data manifestations.

\subsection{Statistical Methods}

Data analysis was processed using SPSS21.0, in which measurement data was expressed in " $(x \pm s)$ ", t-test; count data was expressed in ( $\mathrm{n} \%), \chi^{2}$ test: standard for statistically significant difference: $P<0.05$.

\section{Results}

\subsection{Pathological Diagnosis Results}

Among the 50 patients, there were 19 cases of small liver cancer with 21 lesions detected, 31 cases of massive liver cancer with 36 lesions detected, a total of 57 lesions were detected. Distribution of lesions: (1) 4 lesions less than $1 \mathrm{~cm}, 2$ located in the right anterior lobe of the liver, 2 located in the right posterior lobe of the liver; (2) 17 lesions of 1 to $3 \mathrm{~cm}$ in size, 2 located in the left outer lobe of the liver, 3 located in the left inner lobe of the liver, 4 in the right anterior lobe of the liver, and 8 in the right posterior lobe of the liver; (3) 24 lesions are $3 \sim 5 \mathrm{~cm}$ in size, 4 in the left outer lobe of the liver, 4 in the left inner lobe of the liver, 5 in the right anterior lobe of the liver, and 11 in the right posterior lobe of the liver; 
(4) There are 12 lesions larger than $5 \mathrm{~cm}, 1$ in the left outer lobe of the liver, 3 in the left inner lobe of the liver, 2 in the right anterior lobe of the liver, and 6 in the right posterior lobe of the liver.

\subsection{CT and MRI Lesion Detection}

The detection rate of CT in the portal venous phase was higher than that of MRI, which was statistically significant $(P<0.05)$. The differences in the arterial phase and delayed phase were not statistically significant $(P>0.05)$, see Table 1 .

Table 1. Comparison of CT and MRI Lesion Detection $[n(\%)]$

\begin{tabular}{ccccc}
\hline Method & No. of Lesions & Arterial Phase & Portal Venous Phase & Delayed phase \\
\hline CT & 57 & $50(87.71)$ & $45(78.94)$ & $46(80.70)$ \\
MRI & 57 & $55(96.49)$ & $28(49.12)$ & $47(82.45)$ \\
$\chi^{2}$ & $/$ & 3.015 & 11.007 & 0.058 \\
$P$ & $/$ & $>0.05$ & $<0.05$ & $>0.05$ \\
\hline
\end{tabular}

\subsection{Diagnosis Accuracy}

Among the 57 lesions, the diagnostic accuracy of enhanced CT scan was $85.96 \%$ (49/57), and the diagnostic accuracy of enhanced MRI scan was 91.11\% (52/57). The difference comparison was $\chi^{2}=$ $0.781, P=0.376$.

\subsection{Imaging Data Manifestations}

After enhanced CT and MRI scanning of primary liver cancer, the portal venous phase and the delayed phase lesions show iso-density or low-density, with the typical "fast-in fast-out" characteristics, see Table 2.

Table 2. Enhanced Features of Primary Liver Cancer in Each Phase by CT and MRI $[n(\%)]$

\begin{tabular}{cccccccc}
\hline Method & No. of Lesions & High-Iso-Iso & High-Low-Low & High-Iso-Low & High-High-High & Iso-Low-Iso & Low-Low-Low \\
\hline CT & 57 & $5(8.77)$ & $36(63.16)$ & $5(8.77)$ & $3(5.26)$ & $5(8.77)$ & $3(5.26)$ \\
MRI & 57 & $5(8.77)$ & $17(29.82)$ & $23(40.35)$ & $5(8.77)$ & $4(7.02)$ & $3(5.26)$ \\
$\chi^{2}$ & $/$ & - & 14.255 & 14.886 & 0.538 & 0.121 & - \\
$P$ & 1 & - & $<0.05$ & $<0.05$ & $>0.05$ & $>0.05$ & - \\
\hline
\end{tabular}

2.5 The Display of the Primary Liver Cancer Lesion Capsules by CT and MRI Scan

MRI scan showed the primary liver cancer lesion capsules better than $\mathrm{CT}$, but the difference was not statistically significant $(P>0.05)$. See Table 3:

Table 3. The Display of the Primary Liver Cancer Lesion Capsules by CT and MRI Scan $[n(\%)]$

\begin{tabular}{ccccc}
\hline Method & No. of Lesions & $\begin{array}{c}\text { No Significant } \\
\text { Enhancement }\end{array}$ & $\begin{array}{c}\text { Incomplete Ring } \\
\text { Enhancement }\end{array}$ & $\begin{array}{c}\text { Complete Ring } \\
\text { Enhancement }\end{array}$ \\
\hline CT & 57 & $26(45.61)$ & $8(14.04)$ & $23(40.35)$ \\
MRI & 57 & $22(38.60)$ & $10(17.54)$ & $25(43.86)$ \\
$\chi^{2}$ & $/$ & 0.576 & 0.264 & 0.144 \\
$P$ & $/$ & $>0.05$ & $>0.05$ & $>0.05$ \\
\hline
\end{tabular}

\section{Discussions}

Modern clinical studies point out that liver cirrhosis is closely related to the occurrence of liver cancer. Meanwhile, the surgical treatment of early primary liver cancer is more effective than the treatment of advanced primary liver cancer. Based on this, improving the early diagnosis of primary liver cancer and detection of lesions are conducive to carrying out treatment as soon as possible.

CT and MRI are the main methods for the diagnosis of primary liver cancer. The development of multi-phase dynamic enhanced scanning technology has effectively increased the detection rate of lesions and laid the foundation for further diagnosis and treatment of the disease. In the multiphase enhanced scan, the typical features of primary liver cancer lesions are: significant enhancement in the arterial phase, with high density and high signal characteristics, normal liver parenchyma has no enhancement, or only mild intensity enhancement; portal venous lesions showed low or iso-density signals, and the enhancement of liver parenchyma reached the peak; liver cancer lesions turned into low-density signals in the delayed phase, which also reflected the "rapid rise and fall" enhancement features of primary liver cancer lesions. In this study, patients with primary liver cancer were diagnosed 
with multi-slice spiral CT and enhanced MRI scan. The results showed that the detection rate of enhanced MRI scan was lower than that of the multi-slice spiral CT for portal lesions, but the differences were smaller in the arterial phase and the delayed phase. Meanwhile, the diagnostic accuracy of enhanced MRI scan is slightly higher than that of multi-slice spiral CT, but the difference is small, indicating that the diagnostic accuracy of enhanced MRI scan is slightly higher, which is consistent with the research conclusions of scholars such as Liu Xin et al ${ }^{[2]}$.

MRI has a high resolution for soft tissues and the ability to determine histological characteristics. Combined with dynamic enhanced scanning, it can dynamically reflect the blood supply characteristics of the lesion and distinguish the nature of the lesion based on hemodynamics. Combining the enhancement type and methods, it is easier to identify atypical small cysts and small liver cancers that may be confused by CT scans. In addition, studies have found that one of the pathological features of primary liver cancer is the presence of different degrees of pseudocapsules, which are mostly double-layered, with the inner layer of fibrous tissues and the outer layer of new bile ducts or compressed small blood vessels. In the early stage of dynamic enhancement, the capsules do not get enhanced. After entering the late stage, the density of microvessels in the capsules will increase, resulting in squeezing around the lesion, which in turn causes the sinusoids in the liver parenchyma to be compressed, prolonging the residence time and diffusion time of the contrast agent. At this time, the contrast agent has been discharged from the lesion, so the capsules appear to be irregular and high-density. Based on this, for patients with atypical enhancement, discovering the characteristics of their capsules is helpful for making a clinical diagnosis of primary liver cancer. This study shows that compared with CT, MRI shows better features of pseudocapsule enhancement in primary liver cancer.

In conclusion, both multi-slice spiral CT and MRIenhanced scan can be used for the effective diagnosis of primary liver cancer intrahepatic lesions, and the diagnostic value of the two is equivalent, but the enhanced MRI scan has a slightly higher diagnostic accuracy and can be used as the preferred method.

\section{References}

[1] Sun Y. Value of multi-slice spiral CT in the diagnosis of liver hemangioma and liver tumor [J]. Clinical Research and Practice, 2018, 3(13): 130-131.

[2] Liu X, Zhang Y, Zhang WY, et al. Imaging features of hepatocellular carcinoma by multiple row spiral CT and MRI enhanced scan [J]. Journal of Practical Hepatology, 2018, 21(4): 513-516. 